\title{
Field of moduli and field of definition for curves of genus 2
}

\author{
Gabriel Cardona $^{1}$ \\ Dept. Ciències Matemàtiques i Inf., Universitat de les Illes Balears, \\ Ed. Anselm Turmeda, Campus UIB. \\ Carretera Valldemossa, km. 7.5, \\ 07071 - Palma de Mallorca, Spain. \\ Jordi Quer ${ }^{1}$ \\ Dept. Matemàtica Aplicada II, Universitat Politècnica de Catalunya, \\ Campus Nord, Ed. A0, Desp. 202. \\ Jordi Girona, 1-3, \\ 08034 - Barcelona, Spain
}

\begin{abstract}
Let $\mathcal{M}_{2}$ be the moduli space that classifies genus 2 curves. If a curve $C$ is defined over a field $k$, the corresponding moduli point $P=[C] \in \mathcal{M}_{2}$ is defined over $k$. In [7], Mestre solves the converse problem for curves with $\operatorname{Aut}(C) \simeq C_{2}$. Given a moduli point defined over $k$, Mestre finds an obstruction to the existence of a corresponding curve defined over $k$, that is an element in $\mathrm{Br}_{2}(k)$ not always trivial. In this paper we prove that for all the other possibilities of $\operatorname{Aut}(C)$, every moduli point defined over $k$ is represented by a curve defined over $k$. We also give an explicit construction of such a curve in terms of the coordinates of the moduli point.
\end{abstract}

Key words: genus 2 curves, moduli space, field of moduli, field of definition

\section{Preliminaries on genus 2 curves}

Curves of genus 2, sextic forms and invariants. Let us fix $k$ a perfect field of characteristic char $k \neq 2, \bar{k}$ an algebraic closure, and $G_{k}=\operatorname{Gal}(\bar{k} / k)$

Email addresses: gabriel.cardona@uib.es (Gabriel Cardona), quer@ma2 .upc .es (Jordi Quer).

1 Supported by BFM2000-0794-C02-02 and HPRN-CT-2000-00114 
the absolute Galois group of $k$. If $C / k$ is a genus 2 curve, then it always admits an affine model

$$
y^{2}=f(x)
$$

where $f(x) \in k[x]$ is a polynomial of degree 5 or 6 without multiple roots. We will call this model a hyperelliptic model for $C$.

The classification of genus 2 curves up to isomorphism was performed by Clebsch and Bolza (cf. [4] and [1]) using the classification of binary sextic forms up to linear equivalence. Given a genus 2 curve with hyperelliptic model $y^{2}=f(x)$, the associated sextic form is given by

$$
F\left(x_{1}, x_{2}\right)=x_{2}^{6} f\left(x_{1} / x_{2}\right)
$$

The classification of binary sextic forms uses Hilbert's theory of algebraic invariants (cf. [5]). An invariant is a polynomial expression $I \in k\left[a_{0}, \ldots, a_{6}\right]$ in the coefficients of a generic sextic form

$$
F\left(x_{1}, x_{2}\right)=a_{0} x_{1}^{6}+a_{1} x_{1}^{5} x_{2}+\cdots+a_{5} x_{1} x_{2}^{5}+a_{6} x_{2}^{6},
$$

that under a change of variables

$$
\left(x_{1}, x_{2}\right) \mapsto\left(m x_{1}+n x_{2}, p x_{1}+q x_{2}\right)
$$

associated to $M=\left(\begin{array}{cc}m & n \\ p & q\end{array}\right) \in \mathrm{GL}_{2}(\bar{k})$ changes by a fixed power of $\operatorname{det} M$. Covariants are polynomial expressions in $k\left[a_{0}, \ldots, a_{6}, x_{1}, x_{2}\right]$ with the same property. The homogeneous degree in $x_{i}$ is called the order of the covariant, while the degree in $a_{i}$ is called the degree of the covariant; in particular, invariants are just covariants of order zero. Given an invariant $I$ and a genus 2 curve $C$ or a sextic form $F$, we will denote by $I(C)$ or $I(F)$ the value that takes the invariant when evaluated at this curve or form.

In terms of invariants, the classification goes as follows. Two sextic forms $F$ and $F^{\prime}$ are linearly equivalent if, and only if, there exists $r \in \bar{k}^{*}$ such that for every invariant $I$, one has $I(F)=r^{d} I\left(F^{\prime}\right)$, where $d$ is the degree of $I$. As a consequence, the fact that an invariant annihilates when evaluated to a certain curve depends only on the isomorphism class of the curve.

The algebra generated by the invariants can be generated by five of them, with degrees 2, 4, 6, 10 and 15; this implies that the condition of linear equivalence of forms needs only to be tested for this generating set. Among these generators, there is, up to equivalence, a single algebraic relation which involves terms of degree 30; namely, if $R$ is a generator of degree 15, then this relation states that $R^{2}$ is a polynomial expression in the four generators of even degree. As a consequence, the condition of equivalence needs not to be tested for the 
invariant $R$. Indeed, since the even degree invariants determine $R$ up to sign, after replacing $r$ with $-r$ if necessary, the condition holds for $R$ if it holds for the even degree generators.

Therefore, one can reduce the former condition on linear equivalence to test it only for the four generator invariants of even degree. In the literature appear several such sets of generators: Clebsch invariants, that we will note as $c_{2}, c_{4}, c_{6}, c_{10}$, and are noted $A, B, C, D$ in [4] and [7], Igusa invariants, that we will note as $I_{2}, I_{4}, I_{6}, I_{10}$, and are noted $A, B, C, D$ in [6], and the last set we will mention is that of Igusa arithmetic invariants $J_{2}, J_{4}, J_{6}, J_{10}$ introduced by Igusa in [6]. All these generators have rational coefficients when expressed as polynomials in the coefficients of a generic form. However, while the invariants $J_{i}$ reduce well in any characteristic, the $I_{i}$ fail in characteristics 2 and 3 and the $c_{i}$ in characteristics 2,3 and 5 .

The invariant $I_{10}=2^{12} J_{10}$ is just the discriminant of the form $F$. The forms $F$ corresponding to genus 2 curves are therefore those with $J_{10}(F) \neq 0$.

Absolute invariants and the moduli space. Absolute invariants are quotients of invariants of equal degree. The classification of genus 2 curves up to isomorphism can be stated in terms of absolute invariants: Two curves $C$ and $C^{\prime}$ are $\bar{k}$-isomorphic if, and only if, for every absolute invariant $j$, one has $j(C)=j\left(C^{\prime}\right)$. From this fact, Igusa constructed the variety $\mathcal{M}_{2}$ that classifies genus 2 curves up to isomorphism as a 3 -dimensional affine variety over $\mathbb{Z}$ (cf. $[6])$.

In this moduli space, the classes of curves for which all invariants of degree 2 don't annihilate, which is equivalent to have $J_{2}(C) \neq 0$, form an open set and we can take as coordinates for the moduli point the absolute invariants

$$
j_{1}^{\prime}=\frac{J_{2}^{5}}{J_{10}}, \quad j_{2}^{\prime}=\frac{J_{2}^{3} J_{4}}{J_{10}}, \quad j_{3}^{\prime}=\frac{J_{2}^{2} J_{6}}{J_{10}} .
$$

Similarly, for the set of curves for which all invariants of degree 2 annihilate, but there exists a non-vanishing invariant of degree 4 , that is $J_{2}=0$ and $J_{4} \neq 0$, one can take

$$
j_{1}^{\prime \prime}=0, \quad j_{2}^{\prime \prime}=\frac{J_{4}^{5}}{J_{10}^{2}}, \quad j_{3}^{\prime \prime}=\frac{J_{4} J_{6}}{J_{10}} .
$$

Finally, for the remaining points, one can take

$$
j_{1}^{\prime \prime \prime}=0, \quad j_{2}^{\prime \prime \prime}=0, \quad j_{3}^{\prime \prime \prime}=\frac{J_{6}^{5}}{J_{10}^{3}} .
$$


In must be noted that a point $P=[C] \in \mathcal{M}_{2}$ is defined over a field $k$ if, and only if, its coordinates are in $k$, which amounts to say that all absolute invariants, when particularized to $C$, are in $k$. This way, the set of points on $\mathcal{M}_{2}(k)$ defined over $k$ is in bijection with the set of 3 -tuples $\left(j_{1}, j_{2}, j_{3}\right), j_{i} \in k$, where

$$
j_{i}= \begin{cases}j_{i}^{\prime}, & \text { if } j_{1} \neq 0, \\ j_{i}^{\prime \prime}, & \text { if } j_{1}=0, j_{2} \neq 0, \quad i=1,2,3 . \\ j_{i}^{\prime \prime \prime}, & \text { if } j_{1}=j_{2}=0 .\end{cases}
$$

At first sight, it could seem that to have absolute invariants defined over $k$ does not guarantee that one can find a set of corresponding generator invariants $J_{2}, J_{4}, J_{6}, J_{10}, R \in k$ giving rise to them. The following lemma proves the existence of such invariants.

Lemma 1 For every point $P \in \mathcal{M}_{2}(k)$, there exist invariants defined over $k$ that give the absolute invariants which are coordinates for this point.

PROOF. One can take

$$
\left(J_{2}, J_{4}, J_{6}, J_{10}\right)= \begin{cases}\left(j_{1}, j_{1} j_{2}, j_{1}^{2} j_{3}, j_{1}^{4}\right), & \text { if } j_{1} \neq 0 \\ \left(0, j_{2}, j_{2} j_{3}, j_{2}^{2}\right), & \text { if } j_{1}=0, j_{2} \neq 0 \\ \left(0,0, j_{3}^{2}, j_{3}^{3}\right), & \text { if } j_{1}=j_{2}=0\end{cases}
$$

and verify that with this choices, we get the values for the absolute invariants as defined above.

We can also make a choice in such a way that $R$ is defined over $k$. Since $R^{2}$ can be written as a polynomial expression in the invariants $J_{i}$, we have that $R$ is of the form $R=\sqrt{d} R_{0}$ with $R_{0} \in k$. We can then take $r=\sqrt{d}$ and construct another set of invariats

$$
\begin{aligned}
\left(J_{2}^{\prime}, J_{4}^{\prime}, J_{6}^{\prime}, J_{10}^{\prime}, R^{\prime}\right) & =\left(r^{2} J_{2}, r^{4} J_{4}, r^{6} J_{6}, r^{10} J_{10}, r^{15} R\right) \\
& =\left(d J_{2}, d^{2} J_{4}, d^{3} J_{6}, d^{5} J_{10}, d^{8} R_{0}\right)
\end{aligned}
$$

equivalent to the former and all of them defined over $k$.

Group of automorphisms. In [1], Bolza gives the different possibilities for the reduced group of automorphisms $\operatorname{Aut}^{\prime}(C)=\operatorname{Aut}(C) /\langle\imath\rangle$ of a genus 2 curve, where $\imath$ is the hyperelliptic involution. In [2] the corresponding structures for the full group $\operatorname{Aut}(C)$ are given. If char $k \neq 2,3,5$, the posible automorphism groups of genus 2 curves are

$$
C_{2}, \quad V_{4}, \quad D_{8}, \quad D_{12}, \quad 2 D_{12}, \quad \tilde{S}_{4}, C_{10} .
$$


The generic case corresponds to $\operatorname{Aut}(C) \simeq C_{2}$. The curves having an involution different from the hyperelliptic involution form a surface in $\mathcal{M}_{2}$; the curves with group of automorphisms isomorphic to $D_{8}$ or $D_{12}$ describe two curves in this surface, and they intersect in the single point corresponding to curves with $\operatorname{Aut}(C) \simeq 2 D_{12}$; the curve corresponding to $D_{8}$ has another distinguished point corresponding to the case $\tilde{S}_{4}$. Finally, the curves with group of automorphisms cyclic of order 10 correspond to an isolated point outside the surface of curves with non-hyperelliptic involutions.

The subvariety of curves admitting a non-hyperelliptic involution is characterized by the condition $R=0$, while the single moduli point of curves with group of automorphisms isomorphic to $C_{10}$ is characterized by the condition $J_{2}=J_{4}=J_{6}=0$, that is, $\left(j_{1}, j_{2}, j_{3}\right)=(0,0,0)$.

In characteristic 3 , there is no curve with group of automorphisms isomorphic to $2 D_{12}$. In characteristic 5 , the three isolated points reduce to a single one, for which the reduced group of automorphisms is isomorphic to $\operatorname{PGL}(2,5)$.

Field of moduli and field of definition. Let $C$ be any curve defined over $\bar{k}$. We say that $k$ is a field of moduli for $C$ if $C \simeq{ }^{\sigma} C$ for every $\sigma \in G_{k}$. In the case of genus 2 curves, this condition is equivalent to have the absolute invariants defined over $k$ and, therefore, to say that the point of moduli $P=$ $[C] \in \mathcal{M}_{2}$ is defined over $k$.

If $C$ is defined over $k$, then $P=[C]$ is defined over $k$. The problem we want to solve is the converse: Given a point $P \in \mathcal{M}_{2}(k)$, decide whether there is a curve representing $P$ defined over $k$. In an equivalent formulation, we are interested in the following Galois descent problem: Given a genus 2 curve defined over $\bar{k}$ having $k$ as a field of moduli, decide if there is an isomorphic curve defined over $k$.

The answer to this question is summarized in the following theorem, the first part of which is proved by Mestre in [7],

Theorem 2 Let $P=[C] \in \mathcal{M}_{2}(k)$.

(1) (Mestre) If $\operatorname{Aut}(C) \simeq C_{2}$, there exists an obstruction to the existence of a curve $C^{\prime}$ defined over $k$ and isomorphic to $C$, which is an element in $\mathrm{Br}_{2}(k)$ not necessarily trivial.

(2) If $\operatorname{Aut}(C) \not C_{2}$, there always exists $C^{\prime} / k$ with $C \simeq C^{\prime}$.

The remaining sections of the paper contain the proof of this theorem, split into several cases corresponding to different structures for Aut $(C)$. We remark that all proofs are constructive in the sense that they give an algorithm for constructing a curve defined over $k$ starting from the coordinates of a moduli 
Table 1

Covariants suitable for the case $\operatorname{Aut}(C) \simeq C_{2}$

\begin{tabular}{rlrl}
\hline covariants & order degree \\
\hline$i$ & $=(F, F)_{4}$ & 4 & 2 \\
$\Delta$ & $=(i, i)_{2}$ & & 4 \\
$Y_{1}$ & $=(F, i)_{4}$ & 2 & 3 \\
$Y_{2}$ & $=\left(i, Y_{1}\right)_{2}$ & 2 & 7 \\
$Y_{3}$ & $=\left(i, Y_{2}\right)_{2}$ & 0 & 2 \\
$c_{2}$ & $=(F, F)_{6}$ & 0 & 4 \\
$c_{4}$ & $=(i, i)_{4}$ & 0 & 6 \\
$c_{6}$ & $=(i, \Delta)_{4}$ & 0 & 10 \\
$c_{10}$ & $=\left(Y_{3}, Y_{1}\right)_{2}$ & 2 & 12 \\
$X_{1}$ & $=\left(Y_{2}, Y_{3}\right)_{1}$ & 2 & 10 \\
$X_{2}$ & $=\left(Y_{3}, Y_{1}\right)_{1}$ & 2 & 8 \\
$X_{3}$ & $=\left(Y_{1}, Y_{2}\right)_{1}$ & 0 & $2(i+j+1)$ \\
$A_{i j}$ & $=\left(Y_{i}, Y_{j}\right)_{2}$ & 0 & $2(i+j+k+2)$ \\
$a_{i j k}$ & $=\left(F, Y_{i}\right)_{2}\left(F, Y_{j}\right)_{2}\left(F, Y_{k}\right)_{2}$ & 0 & 15 \\
$R$ & $=-\left(Y_{1}, Y_{2}\right)_{1}\left(Y_{2}, Y_{3}\right)_{1}\left(Y_{3}, Y_{1}\right)_{1}$ & 0 & \\
\hline & & &
\end{tabular}

point $P \in \mathcal{M}_{2}(k)$. In the generic case $\operatorname{Aut}(C) \simeq C_{2}$, this is of course only possible when the obstruction is trivial, and for the construction one needs to find a $k$-rational point on a conic defined over $k$; for all remaining cases an expression for such a curve in terms of invariants is given.

We begin by reviewing the proof of Mestre for $\operatorname{Aut}(C) \simeq C_{2}$ since the way we solve the case $\operatorname{Aut}(C) \simeq V_{4}$ is just a variation of his construction.

\section{The case $\operatorname{Aut}(C) \simeq C_{2}$}

Let $C: y^{2}=f(x)$ be a genus 2 curve defined over $\bar{k}$ with absolute invariants defined over $k$ and $\operatorname{Aut}(C) \simeq C_{2}$. We assume char $k \neq 2,3,5$. Let $F\left(x_{1}, x_{2}\right)=$ $x_{2}^{6} f\left(x_{1} / x_{2}\right)$ be the associated sextic. Mestre considers a set of invariants and covariants defined in Table 1. We refer to [7] for the definition and construction of the covariants $(G, H)_{k}$ obtained from other covariants $G$ and $H$ by means of the so called überschiebung (transvectant) operation. 
These covariants satisfy the relations

$$
\begin{gathered}
\sum_{i, j=1}^{3} A_{i j} X_{i} X_{j}=0 \\
\sum_{i, j, k=1}^{3} a_{i j k} X_{i} X_{j} X_{k}=R^{3} F,
\end{gathered}
$$

which should be understood as identities in $k\left[x_{1}, x_{2}\right]$.

Consider now the conic and cubic defined by the equations

$$
\begin{gathered}
L: \sum_{i, j=1}^{3} A_{i j} Y_{i} Y_{j}, \\
M: \sum_{i, j=1}^{3} a_{i j k} Y_{i} Y_{j} Y_{k} .
\end{gathered}
$$

Since the coefficients $A_{i j}$ and $a_{i j k}$ are invariants of even degree, they can be given as a polynomial expression in the invariants $J_{i}$. Moreover, after lemma 1 , given a moduli point, one can find $J_{i}$ over $k$ that represent the point and, therefore, give equations for $L$ and $M$ with coefficients in $k$. We remark that, in [7], Mestre makes linear changes in these equations to put the coefficients in terms of absulute invariants in order to have the varieties defined over $k$; however, after lemma 1, this is no longer necessary.

In this context, Mestre proves the following theorem.

Theorem 3 With the notations above, there exists a curve of genus 2 defined over $k$ that represents the moduli point if, and only if, $L(k) \neq \emptyset$.

Moreover, if the condition is satisfied, one can find a model for $C$ defined over $k$ as follows. With a rational point $P \in L(k)$ one constructs a $k$-isomorphism

$$
\left(x_{1}, x_{2}\right) \mapsto\left(T_{1}\left(x_{1}, x_{2}\right), T_{2}\left(x_{1}, x_{2}\right), T_{3}\left(x_{1}, x_{2}\right)\right): \mathbb{P}^{1} \rightarrow L .
$$

After replacing $Y_{i}$ with $T_{i}$ in the equation for the cubic $M$, one obtains a sextic form with coefficients on $k$ and with the desired absolute invariants.

Remark 4 This construction works well in every characteristic different from 2, 3 and 5. This is because in the construction of the invariants $A_{i, j}$ and $a_{i, j, k}$ and the covariants $X_{i}$ one introduces denominators that are multiples of these primes. Nevertheless, if one multiplies this covariants and invariants by suitable integers, one gets another set of covariants and invariants with integer coefficients.

With this modifications, the conic and cubic can be defined in every characteristic, and their coefficients can be expressed in terms of the invariants $J_{i}$. 
Table 2

Covariants suitable for the case $\operatorname{Aut}(C) \simeq V_{4}$

\begin{tabular}{|c|c|c|}
\hline \multirow{2}{*}{$\frac{\text { covariants }}{\bar{Y}_{1}=(F, i)_{4}}$} & \multicolumn{2}{|c|}{ order degree } \\
\hline & 2 & 3 \\
\hline $\bar{Y}_{2}=\left(i, Y_{1}\right)_{2}$ & 2 & 5 \\
\hline $\bar{Y}_{3}=\left(Y_{1}, Y_{2}\right)_{2}$ & 2 & 8 \\
\hline $\bar{X}_{1}=\left(\bar{Y}_{2}, \bar{Y}_{3}\right)_{1}$ & 2 & 13 \\
\hline $\bar{X}_{2}=\left(\bar{Y}_{3}, \bar{Y}_{1}\right)_{1}$ & 2 & 11 \\
\hline $\bar{X}_{3}=\left(\bar{Y}_{1}, \bar{Y}_{2}\right)_{1}$ & 2 & 8 \\
\hline $\bar{A}_{i j}=\left(\bar{Y}_{i}, \bar{Y}_{j}\right)_{2}$ & 0 & $2(i+j+1)+\delta_{3}(i, j)$ \\
\hline $\bar{a}_{i j k}=\left(F, \bar{Y}_{i}\right)_{2}\left(F, \bar{Y}_{j}\right)_{2}\left(F, \bar{Y}_{k}\right)_{2}$ & 0 & $2(i+j+k+2)+\delta_{3}(i, j, k)$ \\
\hline $\bar{R}=-\left(\bar{Y}_{1}, \bar{Y}_{2}\right)_{1}\left(\bar{Y}_{2}, \bar{Y}_{3}\right)_{1}\left(\bar{Y}_{3}, \bar{Y}_{1}\right)_{1}$ & 0 & 16 \\
\hline
\end{tabular}

This way, one can make the method of construction work for characteristic 3. However, the conic one obtains is always degenerated in characteristic 5 and therefore this method fails to work in this characteristic.

\section{The case $\operatorname{Aut}(C) \simeq V_{4}$}

Let $C: y^{2}=f(x)$ be a genus 2 curve defined over $\bar{k}$ with absolute invariants defined over $k$ and $\operatorname{Aut}(C) \simeq V_{4}$. As in the previous section we assume char $k \neq$ $2,3,5$, although the remark 4 also holds here.

The conic constructed by Mestre from the absolute invariants of $C$ is in this case degenerated. We mimic the construction of Mestre using another pair of conic and cubic to solve the problem.

Let us introduce another set of invariants and covariants defined by the expressions in Table 2 . In this table, $\delta_{3}$ stands for the number of its arguments equal to 3 . Therefore, the invariants $\bar{A}_{13}, \bar{A}_{23}, \bar{A}_{31}, \bar{A}_{32}$ have odd degree, are multiple of $R$, and annihilate for curves with non-hyperelliptic involutions. Also, the invariants $\bar{a}_{i j k}$ with an odd number of subindexes equal to 3 annihilate for these curves. As for the rest, all of them have even degree and can be expressed as polynomials in the invariants of even degree. We get the following identities expressing the invariants $\bar{A}_{i j}$ and $\bar{a}_{i j k}$ in terms of Clebsch invariants and the $R$ invariant, recalling that a permutation of indexes keeps 
the invariants unaltered:

$$
\begin{aligned}
\bar{A}_{1,1} & =\frac{1}{3}\left(c_{2} c_{4}+6 c_{6}\right), \\
\bar{A}_{1,2} & =\frac{2}{3}\left(c_{4}^{2}+c_{2} c_{6}\right), \\
\bar{A}_{1,3} & =0 \\
\bar{A}_{2,1} & =\frac{2}{3}\left(c_{4}^{2}+c_{2} c_{6}\right), \\
\bar{A}_{2,2} & =c_{10} \\
\bar{A}_{2,3} & =0 \\
\bar{A}_{3,1} & =0 \\
\bar{A}_{3,2} & =0 \\
\bar{A}_{3,3} & =\frac{1}{2 \cdot 3^{2}}\left(-4 c_{4}^{4}-8 c_{2} c_{4}^{2} c_{6}-4 c_{2}^{2} c_{6}^{2}+3 c_{2} c_{4} c_{10}\right)
\end{aligned}
$$

and

$$
\begin{aligned}
\bar{a}_{1,1,1}= & \frac{4}{3^{3} \cdot 5^{2}}\left(c_{2}^{2} c_{6}-6 c_{4} c_{6}+9 c_{10}\right), \\
\bar{a}_{1,1,2}= & \frac{2}{3^{3} \cdot 5^{2}}\left(2 c_{4}^{3}+4 c_{2} c_{4} c_{6}+12 c_{6}^{2}+3 c_{2} c_{10}\right), \\
\bar{a}_{1,1,3}= & \frac{-1}{2 \cdot 3 \cdot 5^{2}} R \\
\bar{a}_{1,2,2}= & \frac{2}{3^{4} \cdot 5^{2}}\left(3 c_{2} c_{4}^{3}+4 c_{2}^{2} c_{4} c_{6}+12 c_{4}^{2} c_{6}+18 c_{2} c_{6}^{2}+9 c_{4} c_{10}\right), \\
\bar{a}_{1,2,3}= & \frac{-1}{2^{2} \cdot 3^{2} \cdot 5^{2}} c_{2} R, \\
\bar{a}_{1,3,3}= & \frac{1}{3^{5} \cdot 5^{2}}\left(-3 c_{2}^{2} c_{4}^{4}+24 c_{4}^{5}-4 c_{2}^{3} c_{4}^{2} c_{6}+42 c_{2} c_{4}^{3} c_{6}+6 c_{2}^{2} c_{4} c_{6}^{2}+72 c_{4}^{2} c_{6}^{2}\right. \\
\bar{a}_{2,2,2}= & \frac{2}{3^{4} \cdot 5^{2}}\left(9 c_{4}^{4}+18 c_{2} c_{4}^{2} c_{6}+8 c_{2}^{2} c_{6}^{2}+6 c_{4} c_{6}^{2}-9 c_{6} c_{10}\right), \\
\bar{a}_{2,2,3}= & \frac{-1}{2^{2} \cdot 3^{2} \cdot 5^{2}} c_{4} R, \\
\bar{a}_{2,3,3}= & \frac{1}{3^{5} \cdot 5^{2}}\left(3 c_{2} c_{4}^{5}+10 c_{2}^{2} c_{4}^{3} c_{6}-6 c_{4}^{4} c_{6}+8 c_{2}^{3} c_{4} c_{6}^{2}+6 c_{2} c_{4}^{2} c_{6}^{2}+24 c_{2}^{2} c_{6}^{3}\right. \\
& \left.-36 c_{4} c_{6}^{3}+18 c_{4}^{2} c_{10}+9 c_{2} c_{4} c_{6} c_{10}-54 c_{6}^{2} c_{10}-27 c_{2} c_{10}^{2}\right), \\
\bar{a}_{3,3,3}= & \frac{1}{2^{3} \cdot 3^{3} \cdot 5^{2}}\left(-3 c_{2} c_{4}^{2}-4 c_{2}^{2} c_{6}+6 c_{4} c_{6}+18 c_{10}\right) R . \\
&
\end{aligned}
$$

For the set of invariants and covariants defined, one has identities analogous to those used by Mestre to define $L$ and $M$. Namely, one has

$$
\sum_{i, j=1}^{3} \bar{A}_{i j} \bar{X}_{i} \bar{X}_{j}=0
$$


and

$$
\sum_{i, j, k=1}^{3} \bar{a}_{i j k} \bar{X}_{i} \bar{X}_{j} \bar{X}_{k}=\bar{R}^{3} F
$$

Let us consider the conic and cubic defined by

$$
\begin{aligned}
& \bar{L}: \sum_{i, j} \bar{A}_{i, j} Y_{i} Y_{j}, \\
& \bar{M}: \sum_{i, j, k} \bar{a}_{i, j, k} Y_{i} Y_{j} Y_{k} .
\end{aligned}
$$

As in the case $\operatorname{Aut}(C) \simeq C_{2}$, one can construct $\bar{L}$ and $\bar{M}$ defined over $k$ from a moduli point defined over $k$.

Lemma 5 With the notations as above, and considering a moduli point corresponding to group of automorphisms $V_{4}$, the conic $\bar{L}$ is non-degenerated.

PROOF. The discriminant of $\bar{L}$ is an invariant; therefore, the fact that it does annihilate or not depends only on the moduli point. We can then suppose that we have a representative for the point with hyperelliptic model

$$
y^{2}=x^{6}+\alpha x^{4}+\beta x^{2}+1
$$

with $\alpha, \beta \in \bar{k}$. The fact that a genus 2 curve with non-hyperelliptic involutions always is isomorphic to some curve of this type was proved by Bolza (cf. [1]).

Computing explicitly the discriminant of $\bar{L}$ constructed from this model over $\bar{k}$ we get a non-zero multiple of

$$
(\alpha-\beta)^{4}\left(\alpha^{2}+\alpha \beta+\beta^{2}\right)^{4}\left(-1125+4 \alpha^{3}+110 \alpha \beta-\alpha^{2} \beta^{2}+4 \beta^{3}\right)^{4} .
$$

It is now easy to check that if any of these terms annihilates, then $\operatorname{Aut}(C)$ contains $D_{8}$ or $D_{12}$.

Theorem 6 Let $C$ be a genus 2 curve with group of automorphisms isomorphic to $V_{4}$ and field of moduli $k$. Then, $C$ is isomorphic to the curve defined over $k$ given by the model

$$
\begin{aligned}
& y^{2}=\left(-\bar{A}_{3,3} \bar{a}_{1,1,1} P_{1}^{3}(x)-3 \bar{A}_{3,3} \bar{a}_{1,1,2} P_{1}^{2}(x) P_{2}(x)\right. \\
&-3 \bar{A}_{3,3} \bar{a}_{1,2,2} P_{1}(x) P_{2}^{2}(x)+3 \bar{A}_{2,2} \bar{a}_{1,3,3} P_{1} P_{3}^{2}(x) \\
&\left.\quad-\bar{A}_{3,3} \bar{a}_{2,2,2} P_{2}^{3}(x)+3 \bar{A}_{2,2} \bar{a}_{2,3,3} P_{2}(x) P_{3}^{2}(x)\right),
\end{aligned}
$$


where

$$
\begin{aligned}
& P_{1}(x)=-2 \bar{A}_{1,2}-2 \bar{A}_{2,2} x \\
& P_{2}(x)=\bar{A}_{1,1}-\bar{A}_{2,2} x^{2} \\
& P_{3}(x)=\bar{A}_{1,1}+2 \bar{A}_{1,2} x+\bar{A}_{2,2} x^{2}
\end{aligned}
$$

whose coefficients can be explicitly obtained from the invariants of $C$.

PROOF. Using the previous construction, one gets a non-degenerated conic with coefficients in $k$. The conic $\bar{L}$ has a point defined on a quadratic extension of $k$; namely,

$$
P=\left(0, \sqrt{-\bar{A}_{3,3}}, \sqrt{\bar{A}_{2,2}}\right)
$$

Using this point, one obtains the following parametrization for the conic

$$
\lambda \mapsto\left(P_{1}(\lambda) \sqrt{-\bar{A}_{3,3}}, P_{2}(\lambda) \sqrt{-\bar{A}_{3,3}}, P_{3}(\lambda) \sqrt{\bar{A}_{2,2}}\right)
$$

where $P_{i}$ are the polynomials given in the statement of the theorem. Using the equation for the cubic $\bar{M}$ with $R=0$, since we are assuming $\operatorname{Aut}(C) \simeq V_{4}$, and the parametrization obtained, one obtains that $C$ is isomorphic to the curve given by $y^{2}=f(x)$, where $f$ is

$$
\begin{aligned}
& \sqrt{-\bar{A}_{3,3}}\left(-\bar{A}_{3,3} \bar{a}_{1,1,1} P_{1}^{3}(x)-3 \bar{A}_{3,3} \bar{a}_{1,1,2} P_{1}^{2}(x) P_{2}(x)\right. \\
& -3 \bar{A}_{3,3} \bar{a}_{1,2,2} P_{1}(x) P_{2}^{2}(x)+3 \bar{A}_{2,2} \bar{a}_{1,3,3} P_{1}(x) P_{3}^{2}(x) \\
& \left.\quad-\bar{A}_{3,3} \bar{a}_{2,2,2} P_{2}^{3}(x)+3 \bar{A}_{2,2} \bar{a}_{2,3,3} P_{2}(x) P_{3}^{2}(x)\right) .
\end{aligned}
$$

Therefore, and up to isomorphism, a model for $C$ defined over $k$ is given by the equation in the theorem.

Remark 7 The pair $\bar{L}$ and $\bar{M}$ can also be used as alternatives to Mestre's $L$ and $M$ in the case $\operatorname{Aut}(C) \simeq C_{2}$ for all moduli points outside the surface detemined by $\bar{R}=0$. However, when $R \neq 0$ the previous formula is not valid, and the construction needs to start with a point $P \in \bar{L}(k)$.

If we construct the conics $L$ and $\bar{L}$ from invariants $c_{i} \in k$ with, also, $R \in k$, then of course the obstructions, as elements in $\mathrm{Br}_{2}(k)$, to find a $k$-rational point on $L$ and on $\bar{L}$ coincide. 
4 The case $\operatorname{Aut}(C) \simeq D_{8}, D_{12}$

These cases have been studied in detail in [3]. Therefore, we only state the results and refer to the mentioned paper for the proof, except for the case of $\operatorname{Aut}(C) \simeq D_{12}$ in characteristic 3, not treated in [3], for which we give the proof here.

Theorem 8 Let $C$ be a genus 2 curve with $\operatorname{Aut}(C) \simeq D_{8}$ and field of moduli $k$. Then, $C$ is $\bar{k}$-isomorphic to the curve defined over $k$

$$
y^{2}=x^{5}+x^{3}+t x
$$

where $t \in k \backslash\{0,1 / 4\}$ is the absolute invariant of $C$ given by

$$
t=t(C)= \begin{cases}1+\frac{J_{4}}{J_{2}^{2}}, & \text { if char } k=5, \\ \frac{-J_{2}^{2}}{J_{4}}, & \text { if char } k=3, \\ \frac{8 c_{6}\left(6 c_{4}-c_{2}^{2}\right)+9 c_{10}}{900 c_{10}}, & \text { if char } k \neq 3,5 .\end{cases}
$$

Theorem 9 Let $C$ be a genus 2 curve with $\operatorname{Aut}(C) \simeq D_{12}$ and field of moduli $k$. If char $k \neq 3$, then $C$ is $\bar{k}$-isomorphic to the curve defined over $k$

$$
y^{2}=x^{6}+x^{3}+t
$$

where $t \in k \backslash\{0,1 / 4\}$ is the absolute invariant of $C$ given by

$$
t=t(C)= \begin{cases}-1-\frac{J_{4}}{J_{2}^{2}}, & \text { if } \operatorname{char} k=5, \\ \frac{3 c_{4} c_{6}-c_{10}}{50 c_{10}}, & \text { if } \operatorname{char} k \neq 3,5 .\end{cases}
$$

If char $k=3$, then $C$ is $\bar{k}$-isomorphic to the curve defined over $k$

$$
y^{2}=\frac{1}{t_{*}} x^{6}+x^{4}+x^{2}+1,
$$

where $t_{*} \in k^{*}$ is such that $t_{*}^{3}=t$ and $t \in k^{*}$ is the absolute invariant given by

$$
t=t(C)=\frac{-J_{2}^{3}}{J_{6}} .
$$

Consider this last char $k=3$ case. In the same way as the proofs in [3], we see that the group $\operatorname{Aut}(C) \subset \mathrm{GL}_{2}(\bar{k})$ isomorphic to $D_{12}$ is $\mathrm{GL}_{2}(\bar{k})$-conjugated to 
the group $\langle U, V\rangle$ generated by

$$
U=\left(\begin{array}{ll}
-1 & 1 \\
-1 & 0
\end{array}\right), \quad V=\left(\begin{array}{ll}
0 & 1 \\
1 & 0
\end{array}\right)
$$

This implies that $C$ is $\bar{k}$-isomorphic to the curve

$$
y^{2}=a_{6} x^{6}+a_{4} x^{4}+\left(a_{4}+2 a_{6}\right) x^{3}+a_{4} x^{2}+a_{6},
$$

where $a_{4}, a_{6} \in \bar{k}^{*}$, because otherwise the polynomial has multiple roots. After changing this curve with the isomorphism associated to the matrix $\sqrt{a_{4}}\left(\begin{array}{ll}1 & 1 \\ 0 & 1\end{array}\right)$, one obtains the isomorphic curve given by the equation

$$
y^{2}=\frac{a_{6}}{a_{4}} x^{6}+x^{4}+x^{2}+1
$$

and $C$ is $\bar{k}$-isomorphic to a the curve with hyperelliptic model

$$
C_{t_{*}}: y^{2}=\frac{1}{t_{*}} x^{6}+x^{4}+x^{2}+1, \quad t_{*}=\frac{a_{6}}{a_{4}} i n \bar{k}^{*}
$$

Computing the absolute invariant of the theorem one obtains that $t\left(C_{t_{*}}\right)=t_{*}^{3}$. Therefore, since we are in characteristic $3, \mathrm{Frob}_{3}$ is a bijection, and it follows that $C_{t_{*}}$ and $C_{t_{*}^{\prime}}$ are isomorphic if, and only if, $t_{*}=t_{*}^{\prime}$. Moreover, if $C$ has $k$ as a field of moduli, then $t=t(C)$ belongs to $k$; since Frob Fis $_{3}$ an automorphism of $k$, we can find $t_{*} \in k$ with $t=t_{*}^{3}$ and $C$ is isomorphic to the curve defined over $k$ with equation

$$
y^{2}=\frac{1}{t_{*}} x^{6}+x^{4}+x^{2}+1
$$

5 The case $\operatorname{Aut}(C) \simeq 2 D_{12}, \tilde{S}_{4}, C_{10}$

The remaining trivial cases correspond to three single points in $\mathcal{M}_{2}$. For the sake of completeness, we give below an explicit equation defined over $k$ for every moduli point.

Theorem 10 Let $C$ be a genus 2 curve with group of automorphisms isomorphic to $2 D_{12}, \tilde{S}_{4}$ or $C_{10}$. Then, $C$ is $\bar{k}$-isomorphic to the curve given by

$$
\begin{array}{ll}
y^{2}=x^{6}-1, & \text { if } \operatorname{Aut}(C) \simeq 2 D_{12}, \\
y^{2}=x^{5}-x, & \text { if } \operatorname{Aut}(C) \simeq \tilde{S}_{4}, \\
y^{2}=x^{5}-1, & \text { if } \operatorname{Aut}(C) \simeq C_{2} \times C_{5} .
\end{array}
$$




\section{References}

[1] Oskar Bolza. On binary sextics with linear transformations into themselves. Amer. J. Math, 10:47-70, 1888.

[2] Gabriel Cardona, Josep González, Joan Carles Lario, and Anna Rio. On curves of genus 2 with Jacobian of GL2-type. Manuscripta Math., 98(1):37-54, 1999.

[3] Gabriel Cardona and Jordi Quer. Curves of genus 2 with group of automorphisms isomorphic to $D_{8}$ or $D_{12}$. Submitted, February 2002.

[4] Alfred Clebsch. Theorie der Binärien Algebraischen Formen. Verlag von B. G. Teubner, Leipzig, 1872.

[5] David Hilbert. Theory of Algebraic Invariants. Cambridge University Press, 1993.

[6] Jun-Ichi Igusa. Arithmetic variety of moduli for genus two. Annals of Mathematics, 72(3):612-649, 1960.

[7] Jean-François Mestre. Construction de courbes de genre 2 à partir de leurs modules. In Effective methods in algebraic geometry (Castiglioncello, 1990), pages 313-334. Birkhäuser Boston, Boston, MA, 1991. 